\title{
Die Bildung von Glykokoll im tierischen Organismus.
}

\author{
Von
}

Emil Abderhalden und Panl Hirsch.

(Aus dem physiologischen Institute der Universität Halle a. S.)

(Der Redaktion zugegangen am 19. März 1912.)

In neuerer Zeit ist von verschiedenen Gesichtspunkten aus der Nachweis geführt worden, daß die tierischen Zellen viel umfassendere und mannigfaltigere Synthesen ausführen können, als man früher angenommen hatte. Es unterliegt keinem Zweifel, daß die weitere Erforschung der synthetischen Fähigkeiten tierischer Gewebe noch ungeahnte Erfolge bringen wird. Unsere ganzen Anschauungen über den Zellstoffwechsel befinden sich zurzeit in beständigem Flusse. Es dürfte wohl kaum mehr zweifelhaft sein, daß der tierische Organismus die Bausteine der Phosphatide und vielleicht auch die der Nucleoproteide von Grund aus aufbauen kann. Auch Zuckerarten werden nach den Erfahrungen am pankreaslosen Hunde und beim Diabetes mellitus aus den bei der Desaminierung von Aminosäuren übrigbleibenden Kohlenstoffketten aufgebaut. Eine bis vor kurzem noch gänzlich unentschiedene Frage war die nach der synthetischen Bildung von Aminosäuren. Eine Neubildung war bis auf die jüngst erfolgte Beobachtung von Fellner ${ }^{1}$ ) nur für das Glykokoll erwiesen. Einerseits konnte durch Fütterungsversuche mit sicher glykokollfreiem Casein es sehr wahrscheinlich gemacht werden, daß die genannte Aminosäure von den tierischen Zellen gebildet werden kann. Dann wurde dieses Problem namentlich an Hand der Hippursäurebildung nach Benzoesäurezufuhr studiert. Schon in einer früheren Mitteilung hat der eine von uns in Gemeinschaft mit Gigon und Strau ${ }^{2}$ ) den Glykokollvorrat von Kaninchen, Katzen und Hühnern festgestellt. Diese Untersuchung sollte die Grundlage zur Entscheidung der Frage abgeben, ob der Vorrat an Glykokoll, den die

1) Hanni Fellner, Über synthetische Bildung von Aminosäuren in der Leber. Mitteil. 4. Bildung von Alanin aus Glykogen. Biochemische Zeitschrift, Bd. 38, S. 414 (1912).

2) E. Abderhalden, A. Gigon und E. Strauss, Über den Vorrat an einigen Aminosäuren bei verschiedenen Tieren, Diese Zeitschrift, Bd. 51, S. 311 (1907). 
einzelnen Tiere besitzen, ausreicht, um das zur Hippursäurebildung notwendige Glykokoll zu liefern.

Die Vorstellung, daß nach Eingabe von Benzoesäure der ganze Eiweißstoffwechsel in der Weise beeinflußt wird, daß vornehmlich solche Proteine abgebaut werden, an deren Aufbau viel Glykokoll beteiligt ist, erscheint a priori sehr gezwungen. Dazu kommt, daß diejenigen Proteine, welche viel Glykokoll besitzen, hauptsächlich zu den Stützsubstanzen gehören. Es ist sehr unwahrscheinlich, daß gerade diese Eiweißkörper, die wohl im allgemeinen einen geringen Anteil am Gesamtstoffwechsel nehmen, zum Abbau kommen, um ihr Glykokoll zur Verfügung zu stellen.

Das ganze Problem mußte einer direkten Prüfung zugänglich sein. Wir schlugen folgenden Weg ein. Kaninchen erhielten bestimmte Mengen von Benzoesäure. Am beweisendsten sind die Versuche, bei denen die Versuchstiere hungerten. Wir bestimmten dann die Menge der ausgeschiedenen Hippursäure und berechneten daraus das ausgeschiedene Glykokoll. Hierbei ist noch zu bemerken, daß Glykokoll noch zu anderen Zwecken im Organismus Verwendung findet, z. B. zur Bildung von Gallensäuren. Mit der Feststellung des mit der Hippursäure ausgeschiedenen Glykokolls ist somit nicht das gesamte gebildete Glykokoll bestimmt. Die Versuchstiere wurden dann hydrolysiert und der Vorrat an Glykokoll festgestellt. Das Glykokoll wurde in Form seines Esters isoliert und als Glykokollesterchlorhydrat abgeschieden. Die erhaltenen Werte sind als Minimalzahlen aufzufassen. Nach Feststellung des einen von uns und Weil ${ }^{1}$ ) müssen die erhaltenen Glykokollmengen verdoppelt werden. Vergleicht man die ausgeschiedenen Glykokollmengen mit den bei der Hydrolyse der Körpersubstanzen erhaltenen und mit zwei multiplizierten Werten, dann ergibt sich, daß ganz beträchtlich mehr an der genannten Aminosäure zur Ausscheidung gelangt ist, als dem schließlich verbleibenden Vorrat entspricht.

Berechnet man aus der ausgeschiedenen Gesamtstickstoffmenge die während der Versuchsdauer umgesetzte Eiweißmenge,

1) E. Abderhalden und A. Weil, Über die bei der Isolierung der Monoaminosäuren mit Hilfe der Estermethode entstehenden Verluste. II. Mitteilung, Diese Zeitschrift, Bd. 77, S. 59 (1912). 
dann käme man zur Erklärung der ausgeschiedenen Glykokollmenge zu der gezwungenen Annahme, daß während der Benzoesäurezufuhr Proteine abgebaut worden sind, die außerordentlich reich an Aminoessigsäure sind. Wir haben bereits oben auf das Unwahrscheinliche einer solchenVorstellung hingewiesen. Addiert man zu dem gefundenen Glykokollvorrat das während der Benzoesäurefütterung in der Hungerperiode ausgeschiedene Glykokoll, dann erhält man Glykokollwerte, die viel höher sind, als der Glykokollvorrat eines normalen Tieres. Vergleiche die folgende Tabelle.

\begin{tabular}{|c|c|c|c|c|}
\hline \multirow[t]{2}{*}{ Nr. } & \multicolumn{2}{|c|}{$\begin{array}{l}\text { Glykokollgehalt } \\
\text { des ganzen Tieres } \\
\text { in } g\end{array}$} & \multirow{2}{*}{$\begin{array}{c}\text { Ausgeschiedenes } \\
\text { Glykokoll } \\
\text { während der Hunger- } \\
\text { periode } \\
\text { in } \mathbf{g} \\
\end{array}$} & \multirow{2}{*}{$\begin{array}{c}\text { Ausgeschiedenes } \\
\text { Glykokoll } \\
+ \text { Glykokollvorrat } \\
\text { in } \mathrm{g}\end{array}$} \\
\hline & Isoliert & \begin{tabular}{|c} 
Berechneter \\
Wert $\left.{ }^{1}\right)$
\end{tabular} & & \\
\hline Versuch III & 5,28 & ca. 10 & 11,41 & 21,41 \\
\hline IV & 5,54 & 11 & 6,60 & 17,60 \\
\hline$\triangleright \quad \mathrm{V}$ & 5,15 & 10 & 8,27 & 18,27 \\
\hline VI & 4,45 & 9 & 6,82 & 15,82 \\
\hline VII & 4,28 & 8,5 & 7,58 & 16,08 \\
\hline$\rightarrow \quad$ VII & 5,01 & 10 & 5,70 & 15,70 \\
\hline Normale $\int \mathrm{I}$ & 5,54 & 11 & - & - \\
\hline Tiere & 5,15 & 10 & - & 一 \\
\hline Hunger- 12 & 4,12 & 8 & - & - \\
\hline tiere II & 4,89 & 10 & - & - \\
\hline II & 5,10 & 10 & - & - \\
\hline
\end{tabular}

Ein Vergleich der beim normalen Tier erhaltenen Glykokollmengen mit dem Glykokollvorrat derjenigen Tiere, die Benzoesäure erhalten hatten, ergibt ohne weiteres, daß die Gesamtmenge an Glykokoll im Verlaufe der Fütterungsperiode im Durchschnitt etwas abgenommen hat, doch ist der Unterschied im Vergleich zu der großen Menge des in Form von Hippursäure ausgeschiedenen Glykokolls sehr gering. Vergleicht man endlich den Glykokollvorrat von Kaninchen, die ohne jede Zufuhr nach 7- resp. 8 tägigem Hungern eingegangen

1) Nach den Erfahrungen von Abderhalden und Weil erreicht die Ausbeute an Glykokoll bei Anwendung der Estermethode ca. 50\% des wahren Wertes. 
waren, mit demjenigen der mit Benzoesäure gefütterten Tiere, dann ergibt sich, daß die Eingabe der genannten aromatischen Säure ohne irgend einen in Betracht kommenden Einfluß auf den gesamten Glykokollvorrat gewesen ist.

Unsere Versuchsresultate ergeben die Tatsache, daß der Organismus des Kaninchens große Mengen von Glykokoll bilden kann. Die Neubildung dieser A minosäure ist auf direktem Wege sicher festgestellt. Erwähnt sei, daß Magnus-Levy') die Möglichkeit betont hat, daß Glykokoll beim Abbau verschiedener Aminosäuren entstehen kann. Uns erscheint die Annahme einer direkten Synthese aus Ammoniak, das den Zellen durch Desaminierung von Aminosäuren, Purinbasen usw. stets reichlich zur Verfügung steht, und einer Kohlenstoff kette - vielleicht Glyoxylsäure oder einer ähnlichen Verbindung - viel wahrscheinlicher. Die neueren Beobachtungen von Knoop, $\left.{ }^{2}\right)$ Embden und Schmitz ${ }^{3}$ ) u. A. stützen die erwähnte Ansicht.

Wir haben die gleiche Frage in der gleichen Weise an Hunden zu entscheiden versucht. Die Resultate waren die gleichen. Es kommt ihnen jedoch kein so überzeugender Wert zu, weil es bei diesen Tieren nicht möglich war, durch Benzoesäure genügend große Mengen von Glykokoll in Form von Hippursäure zu entziehen. Wir verzichten aus diesem Grunde auf die Wiedergabe der umfangreichen in den Jahren 1910 und 1911 durchgeführten Versuche.

Unsere Resultate stehen im Einklang mit den eingangs erwähnten Beobachtungen, die bei der.Fütterung von glykokollfreiem Casein gemacht worden sind; ferner stimmen sie mit

1) A. Magnus-Levy, Über die Neubildung von Glykokoll, Studien zur Hippursäurefrage, Biochem. Zeitschrift, Bd. 6, S. 523-540 (1907).

2) F. Knoop, Über den physiologischen Abbau der Säuren und die Synthese einer Aminosäure im Tierkörper, Diese Zeitschrift, Bd. 67, S. 489 (1910). - F. Knoop und Ernst Kertess, Das Verhalten von $\alpha$-Aminosäuren u. $\alpha$-Ketosäuren im Tierkörper, Ebenda, Bd. 71, S. 252 (1911).

3) G. Embden und E. Schmitz, Über synthetische Bildung von Aminosäuren in der Leber, Biochem. Zeitschrift, Bd. 29, S. 423 (1910). 2. Mitteil., Ebenda, Bd. 38, S. 393 (1912). - Ferner Kura Kondo, III. Mitteil., Die Bildung körperfremder Aminosäuren, Ebenda, Bd. 38, S. 407 (1912).

Hoppe-Seyler's Zeitsehrift f. physiol. Chemie. LXXVIII. 
zahlreichen Beobachtungen über die Hippursäurebildung im tierischen Organismus überein. Die Synthese von Hippursäure nach Eingabe von Benzoesäure ist schon wiederholt als Grundlage zur Entscheidung der Frage nach der Herkunft des Glykokolls gewählt worden. Die von diesen Autoren ${ }^{1}$ ) gemachten

1) Th. Pfeiffer und W. Eber, Beitrag zur Frage über die Bildung der Hippursäure im tierischen Organismus, Landwirtschaftl. Versuchsstation, Bd. 49, S. 97 (1897). - Parker and Graham Jusk, On the maximum production of Hippuric acid in the rabits, Americ. Journal of Physiology, Vol. III, 9, S. 472 (1900). - H. Wi ener, Über den Glykokollvorrat des tierischen Organismus, Prager medizin. Wochenschrift, Bd. 26, Nr. 50 und 51 (1901). - R. Gohn, Zur Frage des Glykokollvorrats im tierischen Organismus, Prager mediz. Wochenschrift, Bd. 27, Nr. 5 (1902). R. Gohn, Zur Frage der Glykokollbildung aus Leucin im tierischen Organismus, Arch. f. exp. Pathol. u. Pharm., Bd. 48, S. 177 (1902). H. Wiener, Zur Frage des Glykokollvorrats im tierischen Organismus, Prager mediz. Wochenschrift, Bd. 27, Nr. 24 (1902). - Th. Pfeiffer, R. Riecke und C. Bloch, Die Muttersubstanzen der im Organismus der Pflanzenfresser erzeugten Hippursäure, Mitteil. des landwirtschaftlichen Instituts der Universität Breslau, Bd. 2, S. 695 (1903). - W. Wi e chow ski, Die Gesetze der Hippursäuresynthese, Hofmeisters Beiträge, Bd. 7, S. 204 (1905). - A. Magnus-Levy, Über die Herkunft des Glykokolls in der Hippursäure, Münchener mediz. Wochenschrift, 1905, Nr. 45. R. Gohn, Zur Frage der Glykokollbildung im tierischen Organismus, Arch. f. exp. Pathol. u. Pharm., Bd. 53, S. 435 (1905). - Th. Brugsch und R. Hirsch, Hippursäuresynthese und Ausscheidung der Benzoesäure beim Hunde, Zeitschrift f. exp. Pathol. u. Therapie, Bd. 3, S. 663 (1906). A. Magnus-Levy, Über die Neubildung von Glykokoll, Studien zur Hippursäurefrage, Biochem. Zeitschrift, Bd. 6, S. 523-40 (1907). J. Lewinski, Über die Grenzen der Hippursäurebildung beim Menschen, Arch. f. exp. Pathol. u. Pharm., Bd. 58,.S. 397 (1908). - J. Lewinski, Über die Grenzen der Hippursäurebildung beim Menschen und die Methodik der Hippursäurebestimmung, Arch. f. exp. Path. u. Pharm., Bd. 61, S. 88 (1909). - R. Gohn, Über den Glykokollvorrat des tierischen Organismus, ein Beitrag zur Frage des intermediären Stoffwechsels, Festschrift zur Feier des 60. Geburtstages von M. Jaffé, S. 321 (1910). - E. Friedmann und H. Tachau, Über die Bildung des Glykokolls im Tierkörper, I. Mitteil., Synthese der Hippursäure in der Kaninchenleber, Biochem. Zeitschrift, Bd. 35, S. 88 (1911). - A. J. Ringer, On the maximum production of Hippuric acid in animals with consideration of the origin of Glycocoll in the animal body, Journ. of biolog. Chem., Bd. 10, S. 327 (1911). - A. A. Epstein und S. Bookman, Studien über die Bildung von Glykokoll im Körper, I., Journal of biolog. Chem., Bd. 10, S. 353 (1911). 
Beobachtungen machen es schon sehr wahrscheinlich, daß das zur Ausscheidung gelangende Glykokoll nicht ausschließlich aus präformierter Aminoessigsäure herstammt. Unsere Versuche erbringen den direkten Beweis für die erwähnte Schlußfolgerung.

\section{Experimenteller Teil.}

Um den Glykokollgehalt zu ermitteln, verarbeiteten wir die ganzen Tiere. Nur das Fell und der Darminhalt kamen nicht zur Verarbeitung. ${ }^{1}$ ) Der übrige Teil wurde in einer Fleischhackmaschine zerkleinert und mit konzentrierter Salzsäure übergossen etwa 3 Wochen in verschlossener Flasche stehen gelassen. Die nun fast ganz flüssig gewordene Masse kochten wir 6 Stunden im Rundkolben auf dem Baboblech am Rückflußkühler. In dem Hydrolysat bestimmten wir nach Kjeldahl den Stickstoffgehalt. Nun wurde im Vakuum zur Trockene verdampft und der Rückstand dreimal mit Alkohol und gasförmiger Salzsäure verestert. Nach der dritten Veresterung füllten wir, bevor wir im Vakuum einengten, mit Alkohol auf ein bestimmtes Volumen auf und bestimmten in einem aliquoten Teil den Stickstoffgehalt. Nun engten wir zum Sirup ein, überschichteten mit etwa 11/2-2 Liter Äther und setzten die Aminosäureester nach Pribram mit Ammoniak in Freiheit. Das sich abscheidende Ammoniumchlorid saugten wir ab und wuschen es tüchtig mit Äther aus. Hat man genügend lange Ammoniak eingeleitet, so läßt sich das Ammoniumchlorid gut absaugen und auswaschen. Nur einmal zeigte es fettige Beschaffenheit, doch bereitete das Auswaschen auch in diesem Falle keine Schwierigkeiten.

Der Äther wurde mit wasserfreiem Magnesiumsulfat getrocknet. Wir dampften dann den Äther im Vakuum bei gewöhnlicher Temperatur ab. Um jeden Esterverlust zu vermeiden, hatten wir zwischen Vorlage und Wasserstrahlluftpumpe eine mit verdünnter Salzsäure gefüllte Saugflasche eingeschaltet. Der abdestillierte Äther wurde mit Salzsäure ausgeschüttelt. Die bei der Destillation zwischen $40-105^{\circ}$ übergehenden Ester wurden mit konzentrierter Salzsäure 2 Stunden am Rückfluß-

1) Vgl. E. Abderhalden, A. Gigon und E. Strauss, Über den Vorrat an einigen Aminosäuren bei verschiedenen Tieren, Diese Zeitschrift, Bd. 51, S. 311 (1907). 
kühler gekocht. Wir füllten auf ein bestimmtes Volumen auf und bestimmten in einem aliquoten Teil der Esterfraktion den Stickstoffgehalt.

Die mit Salzsäure ausgeschüttelte Ätherfraktion, die Salzsäure der Vorlage und schließlich die mit Salzsäure gekochte Esterfraktion verdampften wir im Vakuum zur Trockene.

Durch Ausziehen mit absolutem Alkohol trennten wir in dem Rückstande die Aminosäureesterchlorhydrate vom Chlorammonium. Durch langsames Eindunsten unter möglichstem Ausschluß von Feuchtigkeit erhielten wir Krystallfraktionen von Glykokollesterchlorhydrat. Die Mutterlauge der ersten Abscheidung wurde zur Trockene verdampft, der Rückstand mit absolutem Alkohol übergossen und gasförmige, sorgfältig getrocknete Salzsäure eingeleitet. Die fraktionierte Krystallisation wurde so lange fortgesetzt, bis schließlich nach mehrtägigem Stehen keine Spur von Krystallen mehr sich abschied. Die vereinigten Krystallfraktionen wurden dann aus heißem Alkohol umkrystallisiert. Die Reinheit des isolierten Glykokollesterchlorhydrats wurde durch den Schmelzpunkt und die Stickstoffanalyse festgestellt. Schwierigkeiten bereitete bei der Isolierung des Glykokollesterchlorhydrats die Anwesenheit von Chlorammonium. Nach unseren Erfahrungen ist aus diesem Grunde an Stelle der Ammoniakmethode die Verwendung von Natriumäthylat zur Infreiheitsetzung der Ester in solchen Fällen $z u$ bevorzugen.

Was die Versuche selbst anbetrifft, so ist zu bemerken, daß wir den Harn der in Stoffwechselkäfigen gehaltenen Kaninchen in Porzellanschalen, in denen sich Toluol befand, sammelten. Der innerhalb 24 Stunden gelassene Harn wurde sofort zur Hippursäurebestimmung benutzt. Wir überzeugten uns stets von der Reaktion desselben. Er reagierte stets sauer. ${ }^{1}$ )

Bei den Versuchen III, IV, V und VI verfuhren wir zur Bestimmung der Hippursäure in Anlehnung an die Vorschrift von Bunge und Schmiedeberg, ${ }^{2}$ ) wie folgt:

1) A. van de Velde und B. J. Stokvis, Experimentelle Beiträge zur Frage der Hippursäurezerlegung im lebenden Organismus, Arch. für exp. Path. u. Pharm., Bd. 17, S. 189 (1883).

2) S. Bunge und O.Schmiedeberg, Über die Bildung der Hippursäure, Arch. f. exp. Path. u. Pharm., Bd. 6, S. 233 (1877). 
$50 \mathrm{ccm}$ Harn wurden mit Salzsäure angesäuert und $6 \mathrm{mal}$ mit je $50 \mathrm{ccm}$ Essigäther ausgeschüttelt. Die Essigätherauszüge wurden zur Entfernung aufgenommenen Harnstoffes mit Wasser ausgeschüttelt ${ }^{1}$ ) und dann im Vakuum eingedampft. Der Rückstand wurde in Wasser gelöst, die Lösung im Meßkolben auf $50 \mathrm{ccm}$ aufgefüllt. Nun wurde in zwei Proben von je $10 \mathrm{ccm}$ dieser Lösung der Hippursäuregehalt durch Stickstoffbestimmung nach Kjeldahl festgestellt. Die Werte für Hippursäure und Glykokoll berechneten wir aus den so gewonnenen Hippursäurestickstoffwerten.

In den Versuchen VII und VIII bestimmten wir außerdem an zwei Tagen die Hippursäure in Anlehnung an die Vorschriften von Wiechowski. ${ }^{2}$ ). $50 \mathrm{ccm}$ Harn wurden nach Alkalischmachen mit Natriumcarbonat auf dem Wasserbade zur Trockene verdampft. Der Rückstand wurde mit absolutem Alkohol in einen Meßkolben von $150 \mathrm{ccm}$ gespült und nach Auffüllen 24 Stunden stehen gelassen. $100 \mathrm{ccm}$ des raschfiltrierten alkoholischen Auszuges wurden nach Wiechowskis Vorschrift zum Sirup eingedunstet, der Rückstand in wenig Wasser gelöst und die freie Benzoesäure $3 \mathrm{mal}$ mit je $30 \mathrm{ccm}$ Petroläther im Scheidetrichter extrahiert. Die so behandelte Flüssigkeit wurde dann noch 6 mal mit je $50 \mathrm{ccm}$ Essigäther zur Bestimmung der Hippursäure ausgeschüttelt. Die Benzoesäure stellten wir nach Abdunsten des Petroläthers bei gewöhnlicher Temperatur in der gleichen Weise fest, wie wir es zur Bestimmung der nicht resorbierten Benzoesäure getan haben (vgl. S. 300). Die Hippursäure bestimmten wir gravimetrisch nach Abdunstenlassen des Essigäthers bei gewöhnlicher Temperatur. Die so gewonnene Hippursäure war schon recht rein.

Wir wandten auch bei den Versuchen VII und VIII vergleichsweise die Methode von Bunge und Schmiedeberg, wie

1) F. Blumenthal und A. Braunstein, Über die quantitative Hippursäurebestimmung beim Menschen, Hofmeisters Beiträge, Bd. 3, S. 385 (1903).

F. Blumenthal, Zur Methode der Hippursäurebestimmung, Zeitschrift f. klin. Medizin, Bd. 40, S. 339-44.

2) W. Wiechowski, Die Gesetze der Hippursäuresynthese, Hofmeisters Beiträge, Bd. 7, S. 262 (1906). 
oben angegeben, an, bestimmten jedoch in diesen Versuchen die Hippursäure nach Abdunsten des Essigäthers gravimetrisch. Es zeigte sich, da $\beta$ die auf diese verschiedenen Arten gewonnenen Resultate recht gut zusammen übereinstimmten. Einzig bei Anwendung der von Wiechowski angegebenen Methode erhielten wir keine so guten Ausbeuten, immerhin lassen sich bei genügender Übung Werte erhalten, die den nach Bunge und Schmiedeberg erhaltenen nahe kommen. Uns scheint ihr ein Vorzug gegenüber der altbewährten Methode nicht zuzukommen.

Zur Bestimmung der nicht resorbierten Benzoesäure verfuhren wir, wie folgt. Der Gesamtkot eines Kaninchens wurde 3 Tage im Soxhletapparat mit absolutem Alkohol extrahiert, der Alkoholextrakt nach Zugabe von etwas Natriumcarbonat unter vermindertem Druck zur Trockene verdampft, der Rückstand mit heißem Wasser aufgenommen, filtriert und das Filtrat in einem Meßkolben bis zur Marke aufgefüllt. Ein aliquoter Teil wurde nach Ansäuern mit Phosphorsäure der Wasserdampfdestillation unterworfen. Das Destillat wurde durch ein kleines Filterchen in Normal-Natriumcarbonatlösung tropfen gelassen. Nun wurde mit $10 \mathrm{ccm}$ einer $25 \%$ igen Phosphorsäurelösung angesäuert. Dann wurden 21 Wasser unter normalem Druck durchdestilliert und $5 \mathrm{ccm}$ Normal-Sodalösung vorgelegt. Das Destillat wurde auf dem Dampfbade zur Trockene verdampft, der Rückstand in wenig Wasser gelöst und nach Filtration in einem Meßkolben auf ein bestimmtes Volumen aufgefüllt.

$20 \mathrm{ccm}$ dieser Lösung wurden nach Ansäuern mit verdünnter Salzsäure $3 \mathrm{mal} \mathrm{mit} \mathrm{je} 30 \mathrm{ccm}$ Petroläther ausgeschüttelt. Der Petroläther wurde durch einen trockenen Luftstrom bei gewöhnlicher Temperatur abgedunstet und der sehr geringe Rückstand in 50\% \%igem Alkohol gelöst. Nach derVorschrift von Rem ${ }^{1}$ ) wurden je $10 \mathrm{ccm}$ einer $5 \%$ igen $\mathrm{KJ}$ - und $\mathrm{KJO}_{3}$-Lösung hinzugefügt. Die Benzoesäure wird hierdurch in Monojodbenzoesäure übergeführt und die freiwerdende äquivalente Menge Jod mit Natriumthiosulfat titriert.

1) Eduard Remy, Über die quantitative Bestimmung der Benzoesäure, Apoth.-Ztg., Bd. 26, S. 835 (1911). 
Die Bildung von Glykokoll im tierischen Organismus.

301

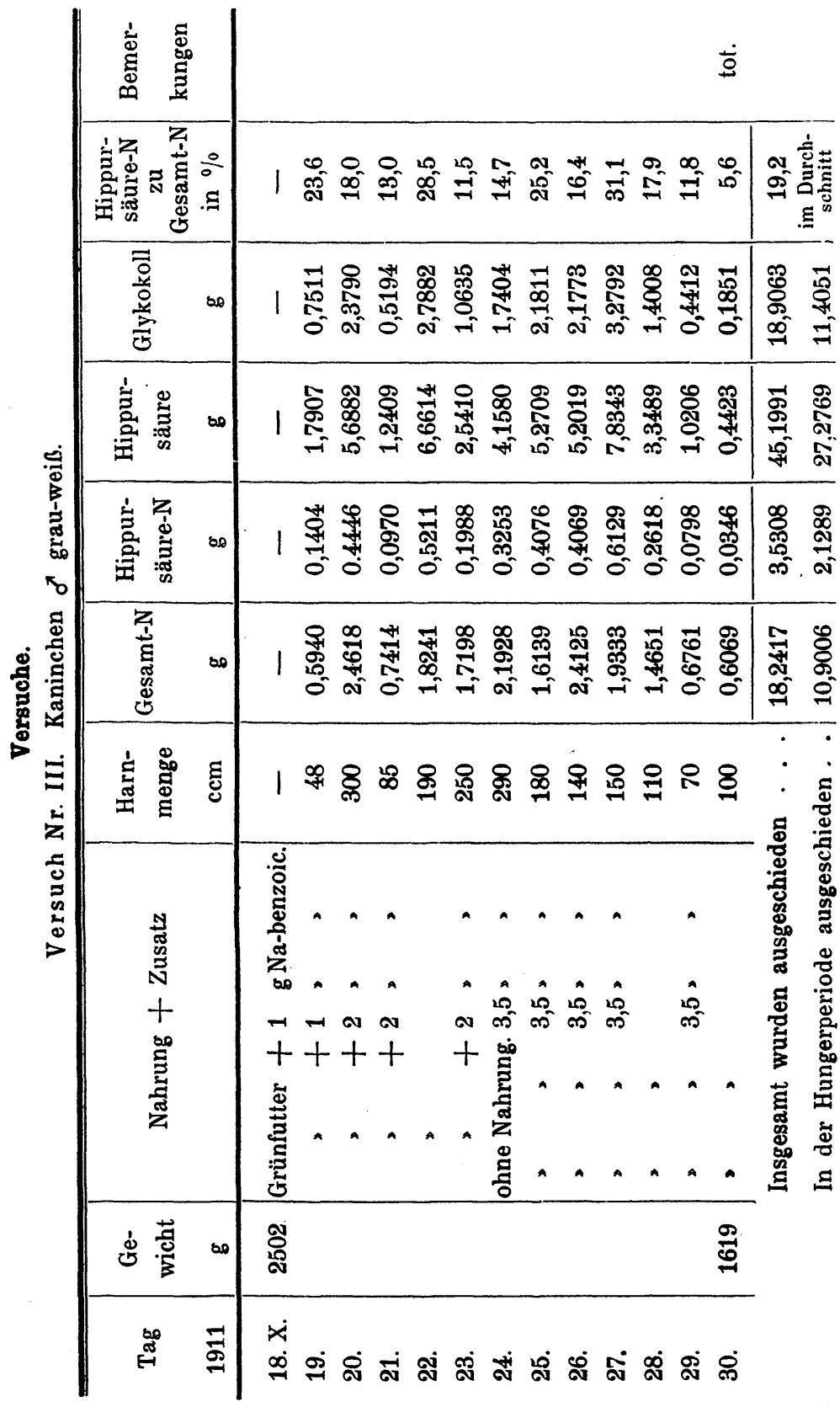




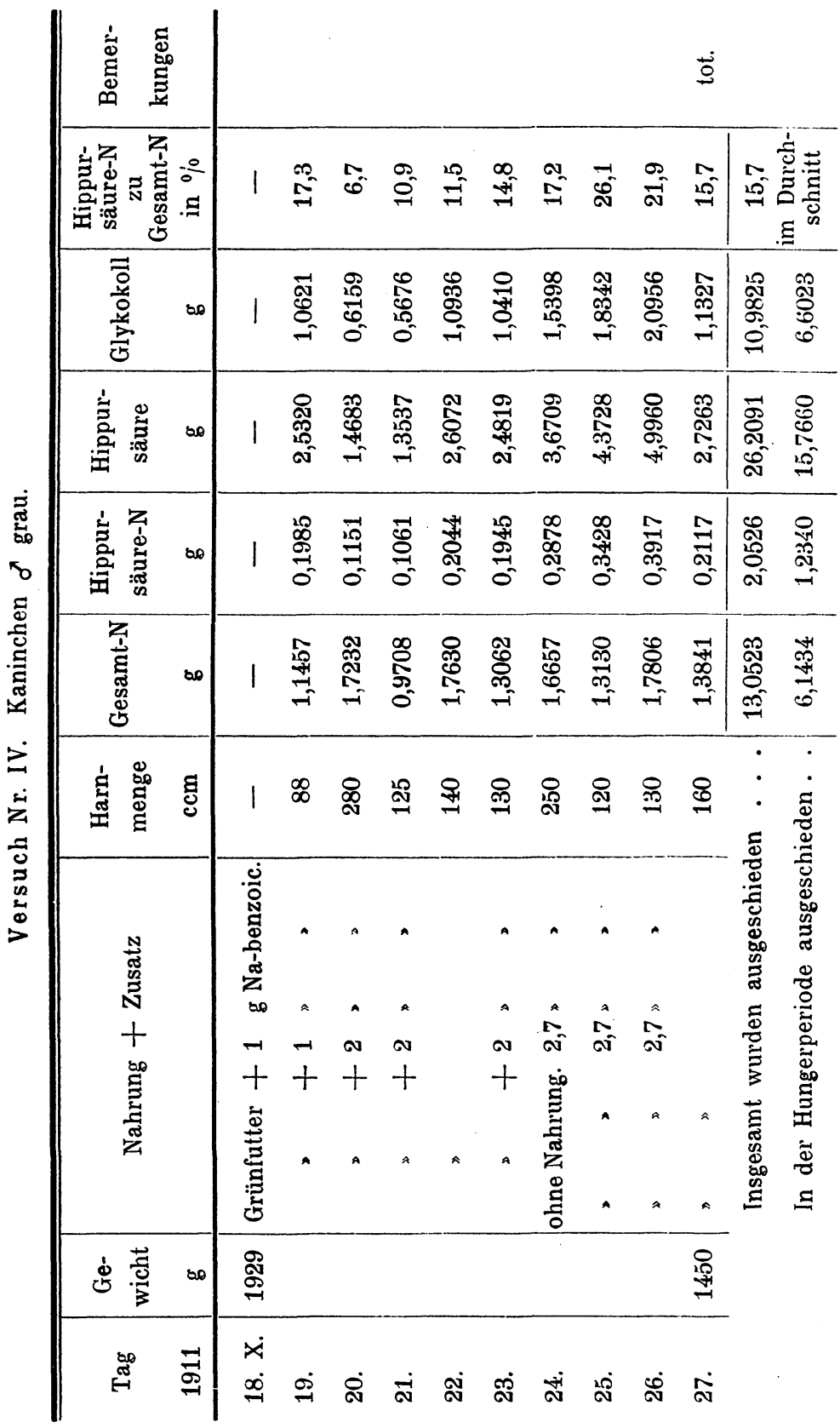


Die Bildung von Glykokoll im tierischen Organismus.

303
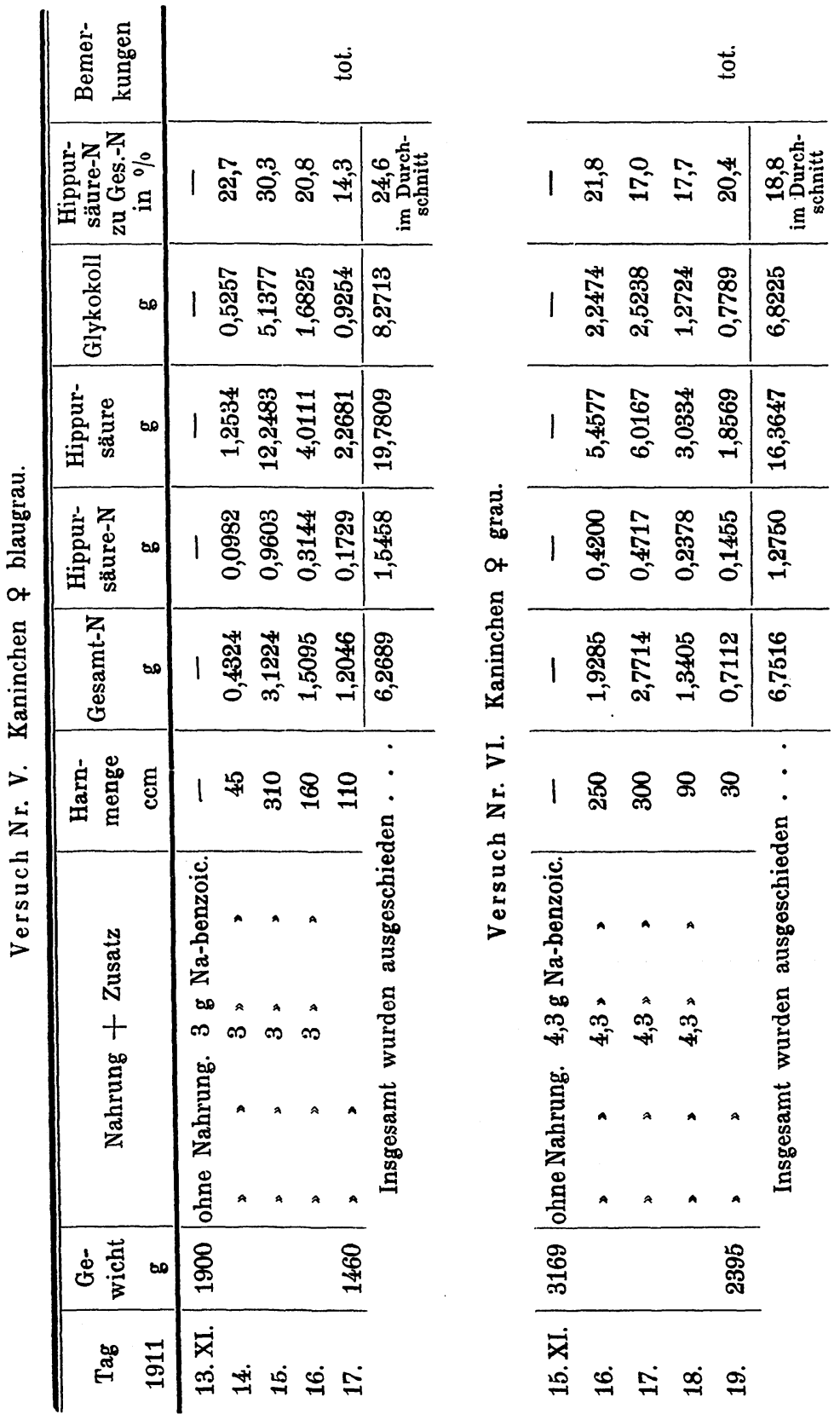


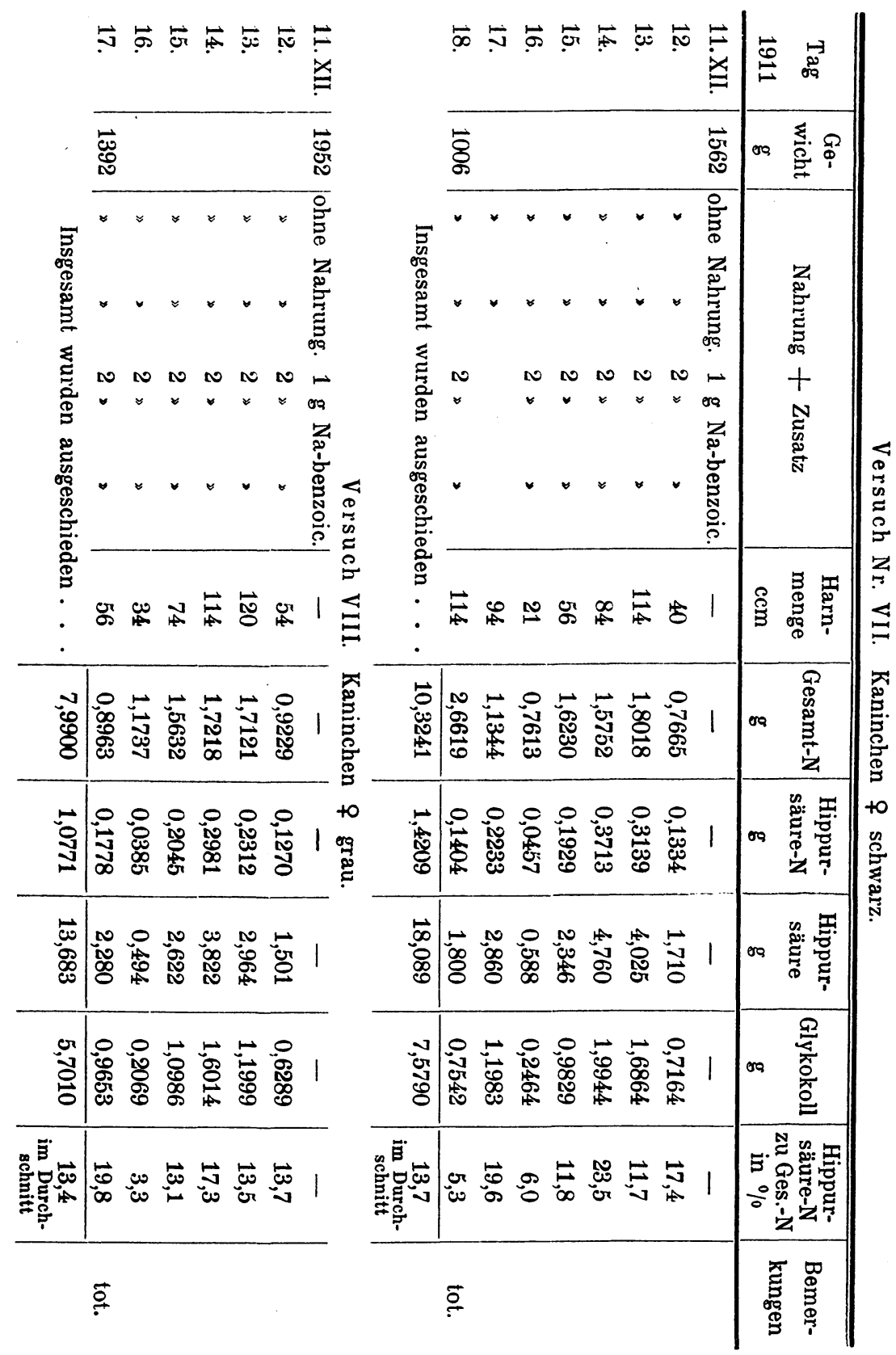


Die Bildung von Glykokoll im tierischen Organismus.

\begin{tabular}{|c|c|c|c|c|c|c|c|c|}
\hline $\mathrm{Nr}$. & & $\begin{array}{c}\text { Ge- } \\
\text { wicht } \\
\text { des } \\
\text { Tieres } \\
\text { g }\end{array}$ & $\begin{array}{c}\text { Gewicht } \\
\text { der } \\
\text { hydroly- } \\
\text { sierten } \\
\text { Menge } \\
\text { g }\end{array}$ & $\begin{array}{c}\mathrm{N} \text {-Gehalt } \\
\text { nach der } \\
\text { Hydro- } \\
\text { lyse } \\
\mathrm{g}\end{array}$ & $\mid \begin{array}{c}\text { N-Gehalt } \\
\text { nach } \\
3 \times \text { Ver- } \\
\text { esterung } \\
\mathrm{g}\end{array}$ & $\begin{array}{c}\text { Ge- } \\
\text { funden } \\
\text { Glyko- } \\
\text { koll } \\
\text { g }\end{array}$ & $\begin{array}{l}\text { Glykokoll } \\
\text { in } \% \text { des } \\
\text { berech- } \\
\text { neten } \\
\text { Eiweiß- } \\
\text { gehaltes }\end{array}$ & $\begin{array}{c}\text { Aus dem } \\
\text { Stickstoff- } \\
\text { gehalt') be- } \\
\text { rechnete } \\
\text { Eiweiß- } \\
\text { menge } \\
\text { g }\end{array}$ \\
\hline ersuch & & 1619 & 1103 & 43,8048 & 37,9080 & 5,28 & & 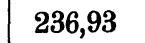 \\
\hline , & & 1450 & 934 & 35,9177 & 31,6181 & 0,04 & & 197,61 \\
\hline & $\mathbf{V}$ & 1460 & 885 & 33,6117 & 30,1579 & 5,15 & 2,73 & 188,49 \\
\hline , & & 2395 & 1540 & 58,8556 & 53,1835 & 4,45 & & 332,40 \\
\hline , & & 1006 & 725 & & 25,3692 & 4,28 & & 158,56 \\
\hline , V & & 1392 & 956 & & 18 & 5,01 & & 89 \\
\hline Normale & & 1949 & 1364 & 44,1315 & 38,5732 & 5,54 & 2,29 & 241,08 \\
\hline Tiere & & 2377 & 1623 & 49,9316 & 48,2504 & 5,15 & 1,70 & 301,57 \\
\hline \multirow{3}{*}{$\begin{array}{c}\text { Hunger- } \\
\text { tiere }\end{array}$} & 2 & 1565 & 984 & 36,1211 & 31,1181 & 4,12 & 2,12 & 194,49 \\
\hline & II & 1610 & 875 & 33,1500 & 30,1225 & 4,89 & 2,59 & 188,27 \\
\hline & & 1845 & 1070 & 40,0572 & 36,2580 & 5,10 & 2,25 & 226,61 \\
\hline
\end{tabular}

Benzoesäurebestimmungen im Kot.

Versuch Nr. III 0,13187 g nicht resorbierte Benzoesäure.

IV 0,24132 g
V 0,34236 g
VI 0,24015 g
VII 0,33523 g
" VIII 0,31041 g

1) Der Berechnung ist der in der alkoholischen Lösung der Aminosäureesterchlorhydrate gefundene Stickstoffwert zugrunde gelegt. 